\title{
Orbiter Capability for Providing Water to the International Space Station according to the Most Probable Flight Attitudes
}

\author{
Brian Dunaway \\ The Boeing Company \\ Marybeth Edeen \\ National Aeronautics and Space Administration
}

\begin{abstract}
Water to be generated by, delivered to, and processed by the International Space Station (ISS) is a critical Environmental Control and Life Support (ECLS) element, especially for the early ISS missions. A significant portion of the water required by the ISS shall be provided by the Shuttle Transportation System (STS) Orbiter. The balance of water generated by the Orbiter Fuel Cells (FC), minus that water consumed by the Orbiter Flash Evaporator System (FES) and crew, is available for transfer to the ISS. During later missions, crew respired and perspired water, as well as effluent water from the Orbiter $\mathrm{LiOH}$ canisters, will be collected as condensate and available for transfer to the ISS.
\end{abstract}

Orbiter radiator performance provides the most variance in determining the amount of net Orbiter water available for transfer to the ISS. As radiator performance decreases, the dependence upon the FES (and FC water) increases for rejecting Orbiter waste heat. Generally, radiator performance decreases as the ISS assembly size increases (especially as solar arrays are added), and also as beta angle increases. ISS solar array deployment necessitates the use of models with articulating solar arrays (for Earth local-vertical attitudes), as array position dramatically affects Orbiter radiator performance. Recent developments in the relaxation of beta angle limitations have also increased the complexity and difficulty of providing water to the ISS. Other factors that may hinder the ability to transfer water are the number of empty Contingency Water Containers (CWCs) available, duration of open-hatch time, crew activity timeline, and full CWC storage capability.

A parametric study has been accomplished that provides a quick-reference table for determining expected water generation rates for ISS missions 2A.2 through 7A.1. An hourly Orbiter water generation rate is reported according to a matrix that consists of: (1) (six) significant changes in ISS assembly configuration; (2) (four) beta angles $\left(0^{\circ},+37^{\circ},+53^{\circ}\right.$, and $\left.+75^{\circ}\right)$; (3) the (three) most representative ISS attitudes (XPOP-0, XPOP-180 and +XVV); (4) (four) Orbiter radiator configurations (both stowed, starboard deployed, port deployed, and both deployed) and (5) the (two) conditions (radiator inlet temperatures and fuel cell power) most consistent with sleep and wake periods. Those permutations of higher probability of occurrence than others have been identified.

Another parametric study has been accomplished that provides a quick-reference table for determining expected water generation rates for ISS assembly complete missions. An hourly Orbiter water generation rate is reported according to a matrix that consists of: (1) (seven) beta angles $\left(-75^{\circ},-60^{\circ},-30^{\circ}, 0^{\circ},+30^{\circ},+60^{\circ}\right.$, and $\left.+75^{\circ}\right)$; (2) the (nine) PYR angles that define the corners of the envelope; (3) (four) Orbiter radiator configurations (both stowed, starboard deployed, port deployed, and both deployed) and (4) the (two) conditions (radiator inlet temperatures and fuel cell power) most consistent with sleep and wake periods. 